\title{
Complicações Intracranianas de Otite Média: A Experiência de 7 Anos do Hospital de Braga
}

\section{Intracranial Complications of Otitis Media: 7 Years of Experience of Hospital de Braga}

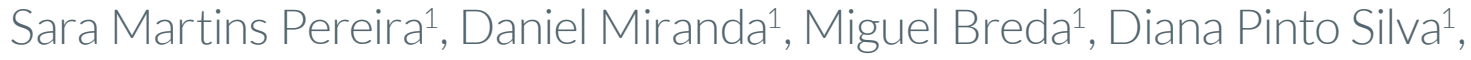
Sérgio Vilarinho ${ }^{1}$, Luís Dias ${ }^{1}$

\section{RESUMO}

INTRODUÇÃO: As complicações intracranianas de otite média representam uma situação relacionada com alta taxa de mortalidade, manifestando-se de forma aguda e constituindo urgências médicas e cirúrgicas. São definidas como disseminação do processo inflamatório/infecioso para além dos espaços pneumatizados do ouvido médio, tendo variadas formas de apresentação.

MATERIAL E MÉTODOS: Consulta dos processos clínicos dos doentes com patologia otológica e patologia intracraniana entre 2008 e 2014 admitidos no Hospital de Braga. Características dos pacientes, diagnósticos otológicos e neurológicos, resultados microbiológicos, abordagem terapêutica e evolução clínica foram os parâmetros analisados. RESULTADOS: Obteve-se um total de 19 doentes, com uma idade média de 40,4 anos. Febre persistente e cefaleias foram os principais sintomas encontrados. Otite média aguda foi o diagnóstico otológico mais associado a complicação intracraniana. Seis pacientes apresentavam mais do que uma complicação, resultando num total de 28 , quase metade das quais correspondendo a meningite.

DISCUSSÃO: Após comparação com estudos semelhantes da literatura, verificamos que os nossos resultados são semelhantes aos já encontrados, exceto no que diz respeito à principal etiologia das complicações intracranianas. CONCLUSÃO: As complicações intracranianas de otite média devem ser consideradas situações de alto risco que requerem intervenção médica imediata.

PALAVRAS-CHAVE: Cefaleia; Encefalopatias; Otite Média/complicações; Meningite

\section{ABSTRACT}

INTRODUCTION: Intracranial complications of otitis media represents a condition related to high mortality, manifesting itself acutely, being medical and surgical emergencies. They are defined as the spread of the inflammatory/infectious process beyond the middle ear space, with various forms of presentation. 
MATERIAL AND METHODS: Consulting the clinical patients' processes with otologic disease and intracranial pathology between 2008 and 2014 treated in Hospital de Braga. Patient characteristics, otologic and neurological disorders, microbiological results, therapeutic approach and outcome were analyzed data.

RESULTS: There was obtained a total of 19 patients with a mean age of 40.4 years. Persistent fever and headache were the main symptoms found. Acute otitis media was the otologic diagnosis more associated with intracranial complication. Six patients had more than one intracranial complication, resulting in a total of 28 , half of them corresponding to meningitis.

DISCUSSION: After comparison with similar studies in the literature, we found that our results are similar to those already found, except with regard to the main cause of intracranial complications.

CONCLUSION: Intracranial complications of otitis media should be considered high-risk situations that require immediate medical intervention.

KEYWORDS: Brain Diseases; Headache; Meningitis; Otitis Media/complications

\section{INTRODUÇÃO}

Apesar da diminuição significativa após o desenvolvimento da terapêutica antibiótica, as complicações intracranianas $(\mathrm{Cl})$ de otite média ainda representam uma situação desafiante relacionada com alta taxa de mortalidade (36\%). ${ }^{1}$ Elas manifestam-se de forma aguda e são urgências médicas e cirúrgicas. São definidas como disseminação do processo inflamatório/infecioso para além dos espaços pneumatizados do ouvido médio e da sua mucosa. ${ }^{2}$ As formas de apresentação mais comuns são meningite, abcesso cerebral, abcesso epidural ou subdural, tromboflebite do seio lateral ou hidrocefalia otítica (quadro de hipertensão intracraniana com origem otológica), que podem decorrer de otite média aguda (OMA) ou otite média crónica (OMC). ${ }^{3} \mathrm{Na}$ fisiopatologia da propagação de processos infeciosos para além dos limites do ouvido médio têm papel: a obstrução do aditus ad antrum, trajetos adquiridos por traumatismo temporal, erosão óssea na sequência de granulação crónica, colesteatoma ou infeção, particularmente por microrganismos mais virulentos, como Haemophilus influenzae, via janelas oval ou redonda para o ouvido interno. ${ }^{2} \mathrm{~A}$ manifestação clínica de uma complicação intracraniana otogénica varia de acordo com a mesma. ${ }^{1}$ No caso da meningite, a apresentação geralmente é evidente, com cefaleias, rigidez da nuca, perda de consciência, agitação, fotofobia, entre outros sintomas. Quando se segue a uma otite média aguda é quase sempre resultado da disseminação hematogénica. Já a meningite associada a otite média crónica resulta habitualmente de uma deiscência da dura-máter que permite disseminação ao líquido cefalorraquidiano da infeção epidural. As restantes complicações têm apresentação menos clara. $O$ abcesso subdural é extremamente raro e mantém-se assintomático até surgirem sinais neurológicos focais ou até mesmo coma. A hidrocefalia otítica apresenta-se caracteris- ticamente por cefaleia, letargia e acentuado papiledema com perturbação da visão. Nos casos de infeção epidural, a região externa ao seio lateral é a mais frequentemente afetada, podendo associar-se a flebite, trombo mural ou a tromboflebite do seio lateral, que em muitos casos se podem manter assintomáticos por longos períodos. Os abcessos cerebrais podem resultar de tromboflebite retrógrada de veias cerebrais ou cerebelosas, tributárias de um seio lateral inflamado, geralmente na sequência de mastoide coalescente, por disseminação pelo canal auditivo interno em casos de labirintite ou em casos de otite média crónica por extensão pelo tégmen para o lobo temporal.

É necessário um elevado índice de suspeição para um diagnóstico precoce e consequentemente tratamento adequado e resultados satisfatórios. ${ }^{1} \mathrm{Na}$ sequência de uma história clínica e exame físico compatível com uma complicação de otite, a imagiologia complementa e esclarece a presença de complicações intracranianas, permitindo apoiar a decisão de realização de exploração cirúrgica que possibilita assim a confirmação do diagnóstico, a colheita de amostras fiáveis para estudo microbiológico e o tratamento do foco inicial. ${ }^{4}$

\section{MÉTODO}

Estudo retrospetivo, em que foram consultados os processos clínicos dos doentes com patologia otológica e patologia intracraniana entre 2008 e 2014 admitidos no Hospital de Braga. Foram excluídos doentes cuja patologia intracraniana não constituísse uma consequência da patologia otológica ou processos clínicos com escassez de dados. Características dos pacientes, diagnósticos otológicos e neurológicos, resultados microbiológicos, abordagem terapêutica e evolução clínica foram os dados analisados. 


\section{RESULTADO}

Obteve-se um total de 19 doentes, 10 do sexo masculino e nove do sexo feminino, com idades compreendidas entre os três e os 82 anos e uma idade média de 40,4 anos. Apenas seis pacientes (31,6\%) apresentavam idade inferior a 18 anos. Os diagnósticos otológicos (Tabela 1) revelaram otite média aguda em 14 casos (73,7\%), otite média crónica colesteatomatosa em três casos $(15,8 \%)$ e otite média crónica simples (OMC) em dois casos (10,5\%).

Em relação ao envolvimento intracraniano, foram encontradas 28 complicações no total, dado que seis doentes apresentavam mais do que uma complicação. Meningite foi detetada em 11 casos (39,29\%), sendo a complicação mais frequente nesta série (Tabela 2). Ainda assim, ocor-

TABELA 1. Diagnósticos otológicos dos doentes com patologia intracraniana associada.

Diagnósticos Otológicos $\quad$ Número de Casos (\%)

\begin{tabular}{|c|c|}
\hline OMA & $14(73,7 \%)$ \\
\hline OMCC & $3(15,8 \%)$ \\
\hline OMC & $2(10,5 \%)$ \\
\hline
\end{tabular}

TABELA 2. Complicações intracranianas.

\begin{tabular}{|l|c|}
\hline Complicações Intracranianas & Número de Casos (\%) \\
\hline Meningite & $11(39,29 \%)$ \\
\hline Cerebrite & $4(14,3 \%)$ \\
\hline Trombose do seio lateral & $4(14,3 \%)$ \\
\hline Empiema epidural & $3(10,7 \%)$ \\
\hline Abcesso cerebral & $3(10,7 \%)$ \\
\hline Abcesso cerebeloso & $2(7,14 \%)$ \\
\hline Hidrocefalia & $1(3,57 \%)$ \\
\hline Total & $\mathbf{2 8 ( 1 0 0 \% )}$ \\
\hline
\end{tabular}

TABELA 3. Resultado das pesquisas

dos agentes patológicos.

\begin{tabular}{|c|c|c|c|}
\hline Culturas & OMA & OMC & OMCC \\
\hline $\begin{array}{l}\text { Negativo ou } \\
\text { não identificado }\end{array}$ & 7 & 2 & 2 \\
\hline Kocurea rosea & 0 & 0 & 1 \\
\hline Pneumococcus & 7 & 0 & 0 \\
\hline
\end{tabular}

TABELA 4. Cirurgias otológicas realizadas em pacientes com patologia intracraniana e respetiva associação com a patologia otológica de base.

\begin{tabular}{|l|c|c|c|c|}
\hline Cirurgia Otológica & OMA & OMC & OMCC & TOTAL \\
\hline $\begin{array}{l}\text { Mastoidectomia } \\
\text { canal wall down }\end{array}$ & 0 & 1 & 2 & 3 \\
\hline $\begin{array}{l}\text { Mastoidectomia canal } \\
\text { wall up (uma das quais } \\
\text { associada a TVT) }\end{array}$ & 2 & 0 & 0 & 2 \\
\hline Aticotomia & 1 & 0 & 0 & 1 \\
\hline TVT isolado & 3 & 0 & 0 & 3 \\
\hline Total & $\mathbf{6}$ & $\mathbf{1}$ & $\mathbf{2}$ & $\mathbf{9}$ \\
\hline
\end{tabular}

reram quatro casos de trombose do seio lateral (14,3\%), quatro casos de cerebrite (14,3\%), três casos de abcesso cerebral (10,7\%), três casos de empiema epidural $(10,7 \%)$, dois casos de abcesso cerebeloso $(7,14 \%)$ e um caso de hidrocefalia (3,57\%).

Verificaram-se cinco casos em que coexistiam complicação intratemporal (otomastoidite) e intracraniana. Febre persistente e cefaleias foram os principais sintomas encontrados. Pneumococcus foi o microrganismo mais envolvido, estando relacionado sobretudo com OMA (Tabela 3).

Foram realizadas intervenções cirúrgicas otológicas (Tabela 4) em nove casos (47,4\% dos doentes), distribuídas da seguinte forma: mastoidectomia canal wall down em 33,3\% ( $n=3)$, mastoidectomia canal wall up em 11,1\% $(n=1)$, aticotomia em 11,1\% ( $n=1)$, mastoidectomia associada a miringotomia com tubo de ventilação transtimpânico (TVT) em 11,1\% (n=1) e miringotomia com TVT em 33,3\% (n=3).

Quanto às intervenções neurocirúrgicas (Tabela 5), foram realizadas em seis pacientes (31,6\%), sendo elas craniotomia em 33,3\% ( $n=2)$, trepanação em 33,3\% ( $n=2)$, shunt ventricular em 16,67\% ( $n=1)$ e drenagem neurocirúrgica via mastoidectomia em 16,67\% (n=1). Dos 19 pacientes, 15 (78,8\%) tiveram um prognóstico favorável. A evolução não foi tão positiva em três (15,8\%) doentes,

\section{TABELA 5. Cirurgias realizadas pela Neurocirurgia em pacientes com patologia intracraniana e respetiva associação com a patologia otológica de base.}

\begin{tabular}{|l|c|c|c|c|}
\hline Neurocirurgia & OMA & OMC & OMCC & TOTAL \\
\hline Craniotomia & 0 & 1 & 1 & 2 \\
\hline Trépano temporal & 0 & 1 & 1 & 2 \\
\hline $\begin{array}{l}\text { Derivação ventricular } \\
\text { externa }\end{array}$ & 1 & 0 & 0 & 1 \\
\hline $\begin{array}{l}\text { Drenagem de } \\
\text { empiema através de } \\
\text { mastoidectomia }\end{array}$ & 1 & 0 & 0 & 1 \\
\hline Total & 2 & 2 & 2 & 6 \\
\hline
\end{tabular}

TABELA 6. Sequelas neurológicas de acordo com a patologia otológica de base.

\begin{tabular}{|l|c|c|c|c|}
\hline $\begin{array}{l}\text { Sequelas } \\
\text { Neurológicas }\end{array}$ & OMA & OMC & OMCC & TOTAL \\
\hline $\begin{array}{l}\text { Sem sequelas } \\
\begin{array}{l}\text { Hemiparesia } \\
\text { esquerda }\end{array}\end{array}$ & 12 & 1 & 2 & $15(78,8 \%)$ \\
\hline Tetraparesia flácida & 1 & 0 & 0 & $1(5,3 \%)$ \\
\hline $\begin{array}{l}\text { Défice de memória } \\
\text { e atenção }\end{array}$ & 1 & 0 & 0 & $1(5,3 \%)$ \\
\hline Morte & 0 & 1 & 0 & $1(5,3 \%)$ \\
\hline Total & 14 & 2 & 3 & $19(100 \%)$ \\
\hline
\end{tabular}


que apresentaram sequelas neurológicas como hemiparesia esquerda, tetraparesia flácida e défice de memória e atenção. Apenas um paciente faleceu (5,3\%) (Tabela 6).

\section{DISCUSSÃO}

Após a análise da literatura, constatamos a evidência amplamente aceite de que o uso de antibioterapia no tratamento da otite média aguda e agudizações de otite média crónica diminuiu drasticamente o número de todas as complicações derivadas de infeções do ouvido médio. Estudos indicam que a incidência de $\mathrm{Cl}$ é de cerca de 0,13 a 1,97\%. ${ }^{4}$ Estudos com grandes séries de complicações são provenientes de países pouco desenvolvidos e provavelmente relacionados com cuidados médicos deficitários, dificuldade de acesso aos mesmos e custo elevado dos antibióticos apropriados., ${ }^{3,4}$ Outro achado comum de várias séries consiste em desenvolvimento de $\mathrm{Cl}$ em idades precoces, especialmente na primeira e segunda década de vida. No estudo de Penido $N$ et al, verificou-se que $26,9 \%$ dos pacientes teria uma idade inferior a 25 anos, ${ }^{4}$ assim como no nosso estudo em que 31,6\% dos pacientes também apresenta idade inferior a 18 anos.

Em todos os estudos consultados, à exceção de Penido $\mathrm{N}$ et al, o sexo masculino foi o mais prevalente, tal como também se verificou no nosso estudo. ${ }^{1-3,5-9}$

Quando comparamos os estudos no que diz respeito à maior incidência de OMA ou OMC como origem das complicações, os resultados são variados. No nosso estudo, a OMA foi prevalente numa percentagem de $73,7 \%$ contra $15,8 \%$ de OMC colesteatomatosa e 10,5\% OMC não colesteatomatosa. Esses achados foram semelhantes aos encontrados por Leskinen $\mathrm{K}$ et al, ${ }^{5}$ em que se verificou 73,6\% de OMA, 12\% OMC e 8\% colesteatoma. No entanto, a maioria dos estudos demonstra incidência superior de OMC numa proporção de 9:2 em relação a OMA.1,4 A prevalência de colesteatoma é amplamente descrita e considerada um fator predisponente importante para $\mathrm{Cl}$ com uma incidência que varia desde os 78\% aos 100\% dos casos de OMC com complicações. ${ }^{4}$ Apesar de não alcançarmos esses resultados no nosso trabalho, é de salientar que temos três casos com antecedentes de colesteatoma previamente conhecido, dois dos quais intervencionados eletivamente previamente às complicações. Um dos casos é particularmente interessante, correspondendo a colesteatoma congénito bilateral numa doente de 17 anos, que resultou em trombose do seio lateral esquerdo com necessidade de revisão de mastoidectomia e achado intraoperatório de recidiva de colesteatoma. A cirurgia prévia ocorreu três anos antes e consistiu em mastoidectomia canal wall up.
A ocorrência de mais do que uma complicação por paciente também é um achado comum. No nosso estudo seis doentes (31,6\%) apresentavam mais do que uma complicação. Verificamos, assim sendo, a associação entre trombose do seio lateral e empiema epidural; abcesso cerebral e cerebrite; empiema epidural, meningite e cerebrite; meningite, abcesso cerebeloso e cerebrite; meningite e abcesso cerebeloso. Penido $\mathrm{N}$ et al ${ }^{4}$ mostraram uma percentagem de $58 \%$ de pacientes com mais do que uma $\mathrm{Cl}$, assim como Yeohet $\mathrm{al}^{3} \mathrm{com} 44 \%$. Este achado indica a necessidade de estudos de imagem rigorosos assim como necessidade de analisar o líquido cefalorraquidiano sempre que se suspeite de uma $\mathrm{Cl}$.

A complicação intracraniana amplamente aceite como a mais frequente de uma OMA e/ou OMC é a meningite, o que se encontra de acordo com os nossos achados. Vários estudos, no entanto, dividem o protagonismo desta complicação com o abcesso cerebral como no caso de Leskinen et al, Osma et al e Modak et al. ${ }^{5-7}$ Os abcessos cerebrais relacionados com otite média têm quase sempre uma localização adjacente ao osso temporal, quase exclusivamente no lobo temporal e cerebelo. Essa localização característica é indicativa do mecanismo principal da infeção que progride através dos limites ósseos do osso temporal por processos de osteíte e erosão óssea. Essa posição do abcesso permite, num grande número de casos, a drenagem simultânea através de uma abordagem mastóidea, evitando uma craniotomia adicional, ${ }^{4}$ como se verificou num dos nossos casos. A punção lombar para diagnóstico de meningite merece atenção adicional. A possibilidade de existência de mais de uma $\mathrm{Cl}$ é comum e a presença de hipertensão intracraniana ou efeito de massa pode promover herniação encefálica com repercussões que podem pôr em causa a vida. É recomendada que seja realizada punção lombar apenas após exame de imagem. ${ }^{8}$

O tratamento da meningite associado a OMA baseia-se no tratamento médico, associado a miringotomia. Nos casos em que esta se associa a OMC, na maioria dos casos deve ser realizada ampla mastoidectomia com exploração de provável deiscência na dura e sua reparação. ${ }^{1}$

Apesar de pouco frequente na maioria dos estudos consultados, a trombose do seio lateral (TSL) aparece no nosso estudo em 14,3\% dos casos. Já o empiema epidural verificou-se em três casos (10,7\%). Penido et al ${ }^{4}$ admitem, no entanto, que a presença de TSL possa ser subestimada na literatura dado o curso muitas vezes assintomático da patologia bem como o facto de ser identificada com maior rigor por angio-ressonância ou angiografia, estudo que não se efetua por rotina. Tipicamente, a trombose inicia-se no seio sigmoide e propa- 
ga-se para o seio lateral. Segundo Oestreicher et al, ${ }^{8}$ é importante a tomografia computorizada (TC) e a ressonância magnética (RM) para diagnóstico e programação cirúrgica de TSL. O tratamento é algo controverso. Pode incluir miringotomia com tubo de ventilação transtimpânico, antibioterapia endovenosa e mastoidectomia. A hipocoagulação pós-cirúrgica é defendida por alguns autores enquanto outros consideram que deve ser reservada para casos de antecedentes de eventos embólicos ou extensão da trombose além do seio sigmoide. ${ }^{8}$ Nos nossos casos, os pacientes foram hipocoagulados com boa evolução.

Verificou-se a presença de um caso de hidrocefalia otítica no nosso trabalho, ao contrário da maioria dos estudos encontrados, em que esta patologia não se verificou, à exceção de Miura et al, ${ }^{1}$ que apresentaram dois casos. Isso pode refletir a baixa incidência desta complicação bem como a dificuldade de diagnóstico da mesma. ${ }^{4}$

As culturas bacterianas foram negativas em 57,9\% dos nossos casos, à semelhança de outros resultados encontrados. Pneumococcus foi, no entanto, o mais encontrado assim como no estudo de Miura et al. Nos restantes estudos, predominaram diferentes bactérias, preferencialmente o Proteus mirabillis. $\bigcirc$ uso prévio de antibióticos pode explicar a baixa sensibilidade para culturas bacterianas verificada em vários estudos. ${ }^{4}$

Após estabilização da terapêutica clínica e estabilização das condições neurológicas, o tratamento cirúrgico deve ser considerado sobretudo nos casos de OMC. ${ }^{9}$ É amplamente recomendado que os procedimentos otológicos sejam preconizados precocemente, preferencialmente antes ou concomitantemente com intervenção neurocirúrgica. Apenas em alguns casos de otite média aguda o tratamento clínico pode ser suficiente. ${ }^{4,8}$

Nos casos de OMC colesteatomatosa, a técnica aberta foi a preferida na maioria dos estudos, como Penido et al, Yeoh et al e Leskinen et al, ${ }^{3-5}$ assim como no nosso trabalho. Técnicas mais conservadoras são reservadas para OMC não colesteatomatosa, colesteatomas restritos ao ouvido médio e alguns casos de OMA com má evolução apesar da terapêutica médica adequada.

A presença de sequelas neurológicas foi de 15,8\%, comparável com outros casos descritos na literatura com percentagens que variam entre os $11,8 \%$ e os $28 \%$. 4,6 Apenas Modak et al apresentaram uma percentagem de apenas 6\% e Yeoh et al não apresentaram nenhuma sequela na sua série. A incidência de mortalidade foi irrelevante no nosso estudo, de apenas 5,3\%, quando comparado com outros estudos em que se verificaram taxas de mortalidade de 7 a 18\%, 4,6,9 ligeiramente superior no caso de Osma et al, com uma taxa de mortalidade de $26 \%$. Estes achados reforçam a necessidade de dar especial atenção à otite média e suas complicações, dada a elevada probabilidade de desfecho menos favorável.

\section{CONCLUSÃO}

As complicações intracranianas de otite média devem ser consideradas situações de alto risco que requerem intervenção médica imediata. Uma abordagem cirúrgica precoce com drenagem do ouvido médio pode ser essencial para evitar complicações do sistema nervoso central. Uma abordagem multidisciplinar é essencial para o sucesso do tratamento.

CONFLITOS DE INTERESSE: Os autores declaram a inexistência de conflitos de interesse na realização do presente trabalho.

FONTES DE FINANCIAMENTO: Não existem fontes externas de financiamento para a realização deste artigo.

CONFIDENCIALIDADE DOS DADOS: Os autores declaram ter seguido os protocolos do seu centro de trabalho acerca da publicação dos dados de doentes.

PROTEÇÃO DE PESSOAS E ANIMAIS: Os autores declaram que os procedimentos seguidos na elaboração do presente trabalho estão em conformidade com as normas das comissões de investigação clínica e de ética, bem como da declaração de Helsínquia e da Associação Médica Mundial.

\section{REFERÊNCIAS}

1. Miura MS, Krumennauer RC, Lubianca Neto JF. Intracranial complications of chronic suppurative otitis media in children. Rev Bras Otorrinolaringol. 2005;71:639-43.

2. Neely J. Surgery of acute infections and their complications. In: Brackmann D, Shelton C, Arriaga M, editors. Otologic Surgery. Amsterdam: Elsevier; 2001.p. 183-94.

3. Long YT, Mahmud R, Sani A, Saim L. Complications of otitis media requiring surgical intervention. Asian J Surg. 2002;25:1704.

4. Penido N, Borin A, Luiz C, Suguri V, Onishi E, Fukuda Y, et al. Intracranial complications of otitis media: 15 years of experience in 33 patients. Otolaryngol Head Neck Surg. 2005;132:37-42.

5. Leskinen K, Jero J. Acute complications of otitis media in adults. Clin Otolaryngol. 2005;30:511-51.

6. OsmaU, CureogluS, HosogluS. The complications of chronicotitis media: report of 93 cases. J Laryngol Otol. 2000;114:97100.

7. Modak V, Chavan V, Borade R, Kotnis D, Jaiswal SJ. Intracranial complications of otitis media: in retrospect. Indian J Otolaryngol Head Neck Surg. 2005;57:130-5.

8. Oestreicher-Kedem Y, Raveh E, Kornreich L, Popovtzer A, Buller N, Nageris B. Complications of mastoiditis in children at the onset of a new millennium. Ann Otol Rhinol Laryngol. 2005;114:147-52.

9. Yung-Song, Li-Ching, Fei-Peng, Kuan J. The prevalence of chronic otitis media and its complication rates in teenagers and adult patients. Otolaryngol Head Neck Surg. 2009;140:165-70. 